\title{
Molecular Size of Retinal Vascular Leakage Determined by FITC-Dextran Angiography in Patients with Posterior Uveitis
}

\author{
E. G. ATKINSON, * S. JONES, $\uparrow$ B. A. ELliS, D. C. DUMONDE AND E. GRAHAM* \\ London
}

\begin{abstract}
Summary
In order to investigate the selective breakdown of the blood-retinal barrier in posterior uveitis angiograms were performed using fluorescein-conjugated dextrans (FITC-dextrans) of different molecular weights (150-kDa, 20-kDa, and 4-kDa) in two healthy controls and six patients with posterior uveitis, and the results compared with those of conventional fluorescein angiography. The smallest FITC-dextran could not penetrate the healthy blood-retinal barrier. Leakage of FITC-dextrans of all sizes was seen from swollen optic discs; dextrans of 4-kDa and 20-kDa leaked from areas of macula oedema; and retinal new vessels allowed the passage of 4-kDa molecules only. These results indicate that there is a differential breakdown of the bloodretinal barrier according to molecular size in various clinical situations associated with posterior uveitis.
\end{abstract}

Leakage of sodium fluorescein from inflamed retinal vessels is a characteristic angiographic feature of posterior uveitis and results from breakdown of the blood-retinal barrier (BRB) at sites such as the optic disc, macula, and areas of neovascularization. ${ }^{1}$ However, standard fluorescein angiography gives qualitative rather than quantitative information, and, because fluorescein is a small molecule (376-Da), will not differentiate between degrees of BRB breakdown that might allow molecules of different molecular weight access to the retina. Prompted by the finding that $50 \%$ of our retinal vasculitis patients have circulating antibodies to retinal proteins, ${ }^{2}$ which might be pathogenic if able to enter the retina, we set out to measure the size of mol- ecules able to leak from the retinal vessels of such patients.

The glucose polymer dextran, available in a wide range of defined molecular weights, can be conjugated at multiple sites with fluorescein isothiocyanate (FITC) to produce high molecular weight fluorescent molecules which can be used as tracers in vascular permeability studies. ${ }^{3,4}$ Studies using these markers have quantified vascular leakage of the BRB in rats with urethane induced retinopathy, ${ }^{5}$ and primates with experimental posterior uveitis. ${ }^{6}$ We report here for the first time in man a method of using graded FITC-dextrans, after the appropriate toxicological studies, in assessing retinal vascular damage in patients with posterior uveitis.

From: Departments of Immunology, *Medical Ophthalmology and †Pharmacy, United Medical and Dental Schools, St Thomas' Campus, London SE1 7EH.

E. G. Atkinson died on 15 June 1990 in a para-gliding accident in France.

Correspondence to: Dr E. Graham MRCP, Medical Eye Unit, St Thomas' Hospital, Lambeth Palace Road, London SE1 7EH. 


\section{Methods}

Manufacture of FITC-dextrans for injection FITC-dextrans of molecular weights 4-kDa, 20-kDa and 150-kDa (FD-4, FD-20, and FD-150 respectively) were purchased from Sigma Chemical Company Ltd (Poole, Dorset, UK) and dissolved in sterile normal saline at $10 \% \mathrm{w} / \mathrm{v}$. The resulting solutions were sterilized by filtration through $0.2 \mu \mathrm{m}$ filters (Amicon Ltd, Gloucester, UK) before being packaged into sterile glass ampoules and stored at $4^{\circ} \mathrm{C}$. The raw materials were assayed for solubility, chromatographic purity and heavy metal contamination. The finished products had a pH 6.5, were assayed by ultraviolet spectroscopy, and investigated for the presence of foreign protein, ${ }^{7}$ endotoxin (Wickham Laboratories Ltd, Fareham, UK), and sterility (British Pharmacopoeia, 1988). Their acute toxicity to rats (Huntingdon Research Centre, Huntingdon, UK) and chemical stability in solution by chromatographic anlaysis was also determined.

\section{Patient selection}

All experiments were performed with the approval of the West Lambeth Health Authority Ethical Committee and patients gave informed consent to the procedure. Two normal subjects (EGA and SJ) and six patients with posterior uveitis were selected for study (Table I). The patients were chosen for their representative features of active posterior uveitis. No patient had ever suffered an allergic reaction.

\section{Angiographic procedure}

A Zeiss fundus camera with conventional fluorescein filters and maximum flash setting was used for photography. Colour fundus photographs were recorded on Ectachrome 64 film

Table I Characteristics of patients studied

\begin{tabular}{cccl}
\hline Patient & Age & Sex & Diagnosis \\
\hline 1 & 30 & M & Normal \\
2 & 28 & M & Normal \\
3 & 27 & M & Idiopathic RV \\
4 & 42 & M & Behçets disease \\
5 & 45 & M & Idiopathic RV \\
6 & 45 & M & Idiopathic RV \\
7 & 35 & M & Sarcoid \\
8 & 33 & F & Idiopathic RV \\
\hline
\end{tabular}

(Kodak, Hemel Hempstead, England). Angiograms were recorded on TMax P3200 film (Kodak, Rochester, NY, USA) and processed for nine minutes at $29^{\circ} \mathrm{C}$ in TMax developer. The procedure comprised rapid intravenous injection of $10 \mathrm{ml}$ of $10 \%$ FITCdextran administered in the sequence FD-150, FD-20 and FD-4; each injection was separated from the next by 40 minutes. Angiograms were recorded at 0,10 , and 20 minutes after each injection. The procedure was completed by recording a conventional angiogram with $5 \mathrm{ml}$ of $20 \%$ sodium fluorescein $(\mathrm{NaFl})$ (B.P.) on FP4 film (Ilford, Mobberley, UK). Records of the subjects' symptoms, blood pressure, and pulse were made at intervals throughout the procedure. Sequential blood and urine samples were taken to measure FITC-dextran concentration, and for tests of hepatic and renal function.

\section{Results}

Analysis of raw material and finished products

All FITC-dextrans were easily dissolved in saline. The raw materials were found to contain less than $15 \mathrm{ppm}$ w/w of each of the heavy metals $\mathrm{Cd}, \mathrm{Hg}, \mathrm{Sn}, \mathrm{As}, \mathrm{Pb}$ and $\mathrm{Sb}$ by inductively coupled plasma Atomic Emission Spectroscopy (British Pharmacopoeia limit $20 \mathrm{ppm}$ ), and were found to have a single molecular weight peak on gel filtration. Each finished product was found to contain less than 1.0 EU/ml of endotoxin (limit 5.0 EU/ml), no foreign protein and to be sterile. No product was significantly toxic to rats when administered at 18 times the proposed human dose and each was stable in solution for at least a year, showing no evidence of degradation into smaller molecular weight species.

\section{Subject responses to injection}

No subject experienced any symptoms after injection of any of the FITC-dextrans. There were no significant changes in pulse rate or blood pressure and no changes in haematological indices, or renal or hepatic function. No injection caused significant visible skin staining. The FITC-dextrans disappeared from the circulation with a half-life proportional to their molecular weight, with FD-4 
and FD-20 being excreted unchanged into the urine.

\section{FITC-dextran angiography in normal subjects}

Angiograms recorded following injection of FD-4, FD-20 and FD-150 in the two normal subjects demonstrated good vascular detail during the arterio-venous (A-V) phase, which was still leasily visible at 10 minutes after injection (Fig. 1). FD-20 gave angiograms of greatest intensity and FD-150 of least, in parallel with the estimated amount of fluorescein administered (Table II). At ten minutes the dye was still present in the vasculature in all three angiograms but details of both disc and macula were only visible with FD-20. Fluorescent staining of the optic disc did not progress beyond the A-V phase and the macula remained dark throughout. Because the amount of fluorescein administered was small (Table II) the intensity of fluorescence observed by the photographer was low during the A-V phase and barely discernible during the late venous phase. The grain evident on the angiograms resulted from the nature of the film/developer combination used and gave a rather low contrast picture.

\section{FITC-dextran angiography in retinal vasculitis patients}

Overall, the angiographic detail in posterior uveitis patients was less than in normal subjects because of media opacities, in particular vitreous haze. However, sufficient detail was preserved to allow reliable determination of leakage using the processed films. Leakage of FITC-dextrans was assessed at the optic disc, macula and areas of neovascularization.

Optic disc: The optic disc leaked $\mathrm{NaFl}$ in four

Table II Amount of fluorescein administered and molecular size

\begin{tabular}{lccc}
\hline Preparation & $\begin{array}{c}\text { Molecular weight } \\
(\mathrm{Da})\end{array}$ & $\begin{array}{c}\text { Amount } \\
(\mathrm{mg})^{2}\end{array}$ & $\begin{array}{c}\text { EDR } \\
(\mathrm{nm})^{3}\end{array}$ \\
\hline $\mathrm{NaFl}^{1}$ & 376 & 1000 & 0.5 \\
$\mathrm{FD}^{1}$ & 4000 & 8.4 & 1.3 \\
$\mathrm{FD}-20$ & 20000 & 16.7 & 3.2 \\
$\mathrm{FD}-150$ & 150000 & 12.5 & 8.5 \\
\hline
\end{tabular}

${ }^{1}$ Present as the disodium salt

${ }^{2}$ Amount present in $10 \mathrm{ml}$ of a $10 \% \mathrm{w} / \mathrm{v}$ solution

${ }^{3}$ Effective diffusion radius (EDR) estimated in $\mathrm{nm}$ of six patients examined. At ten minutes FD-4 was clearly observed to leak from the optic disc and peripapillary region in three patients (Fig. 2). At this time FD-20 leakage was less obvious but discernible particularly at the inferior pole of the disc in these patients

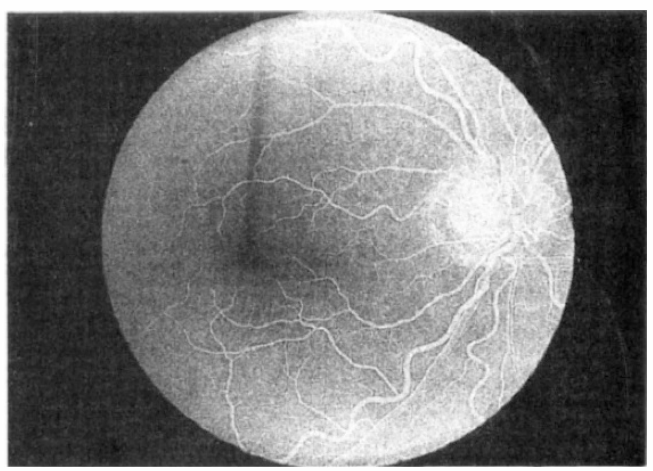

Fig. 1a.

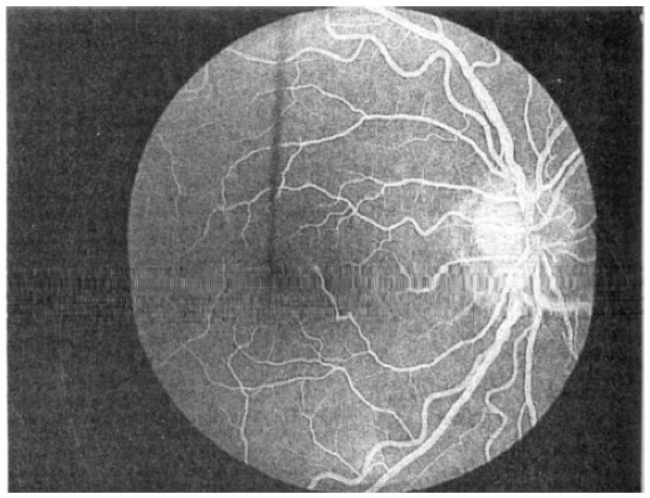

Fig. $1 b$.

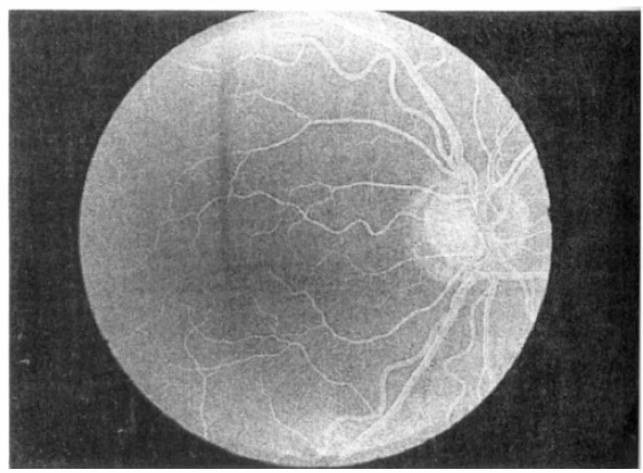

Fig. 1. FITC-dextran angiograms of a normal volunteer. The arteriovenous phases following injections of FD-4 (top), FD-20 (middle), and FD-150 (bottom). Good vascular detail is obtained on each occasion. 

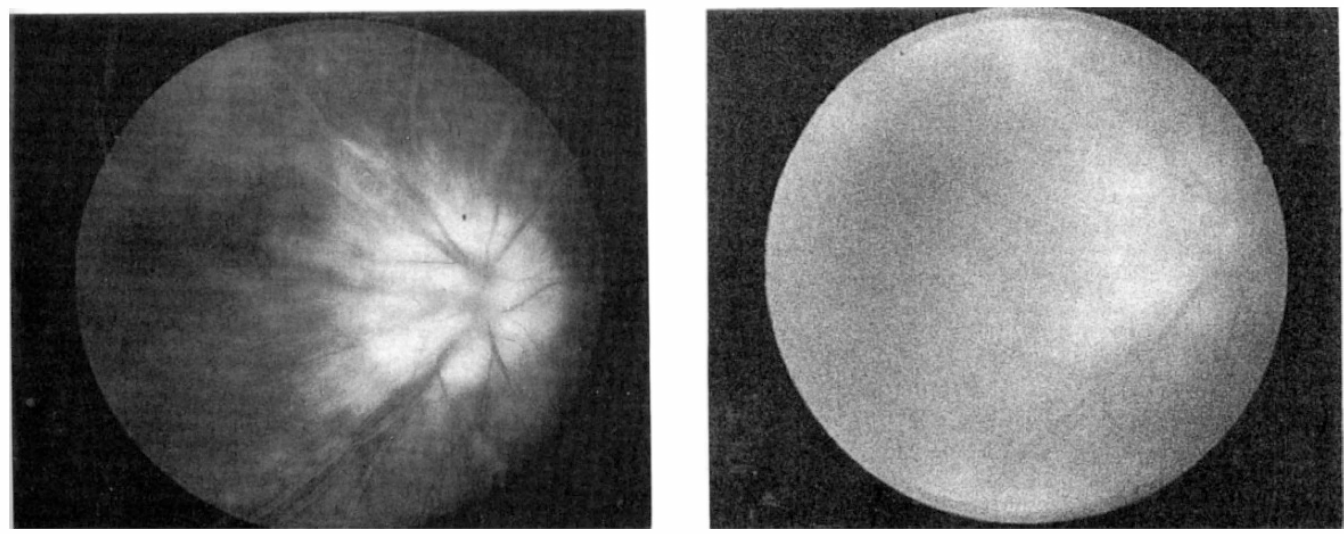

Fig. 2 a

Fig. 2b.

Fig. 2. Fluorescein angiograms in a patient with disc oedema. Figure 2(a) left: 10 minutes after injection of sodium fluorescein angiogram demonstrating leakage from the optic disc and peripapillary region. Figure 2(b) right: 10 minutes after injection of FD-4. The leakage from the optic disc and peripapillary region is still clearly visible.

whereas FD-150 leaked from the optic disc of only two patients and was barely visible at ten minutes. Leakage of FITC-dextrans never extended beyond the boundaries of $\mathrm{NaFl}$ leakage.

Macula: Whereas $\mathrm{NaFl}$ leaked from most areas of the macula in five of the patients studied, the FITC-dextrans leaked in a more selective manner from within the areas of $\mathrm{NaFl}$ leakage (Fig. 3). Both FD-4 and FD-20 leaked in four patients whereas only FD-20 leaked in another, perhaps reflecting its greater fluorescent intensity, although at ten minutes FD-4 was seen more easily than FD-20 which was barely discernible. FD-150 did not leak into any of the maculas studied.

New vessels: FD-4 leaked from retinal new vessels but much more slowly than $\mathrm{NaFl}$ (Fig. 4). Other dextrans showed no leakage.

\section{Discussion}

This study has demonstrated that FITC-dex-

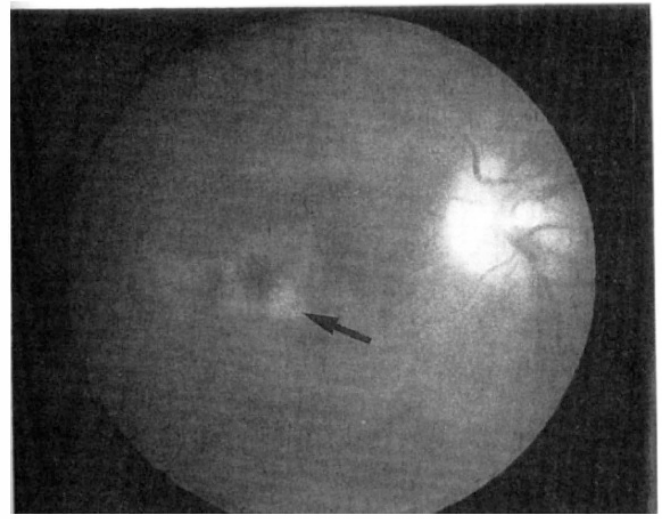

Fig. 3a.

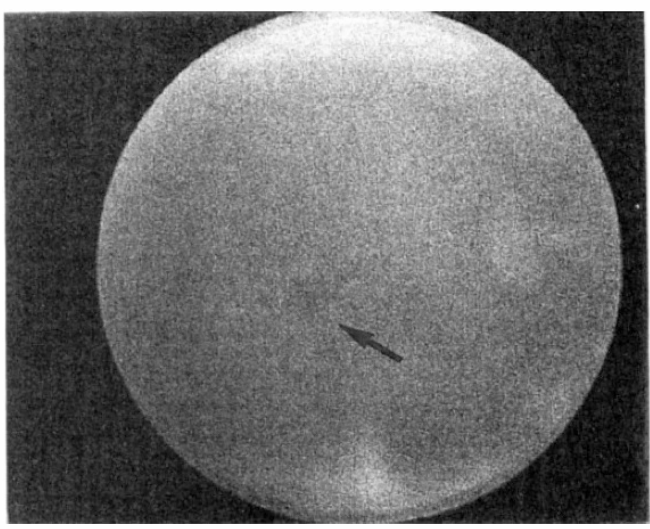

Fig. 3b.

Fig. 3. Fluorescein angiograms in a patient with macular oedema due to sarcoidosis. Figure 3 a (left): 10 minutes after injection of sodium fluorescein. Petalloid macular oedema is seen with some leakage from the optic disc and upper temporal vessels. Figure $3 \mathrm{~b}$ (right): 10 minutes after injection of FD-4. The macular oedema is just visible and the optic disc is clearly seen. (FD-150 angiogram is unavailable for this patient) 


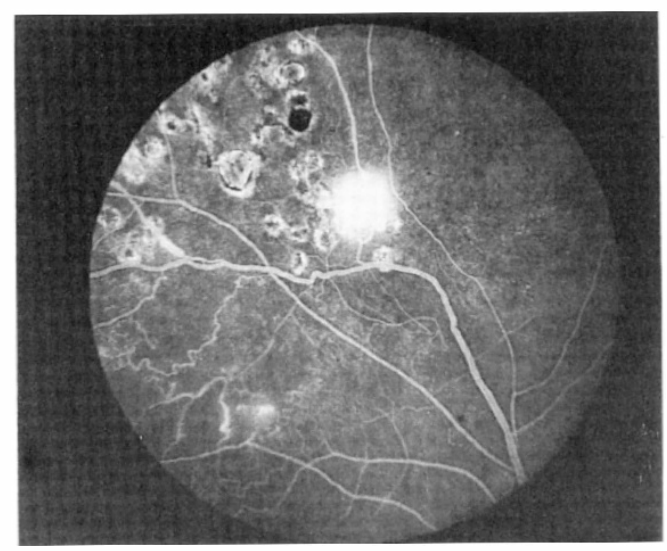

Fig. 4a.

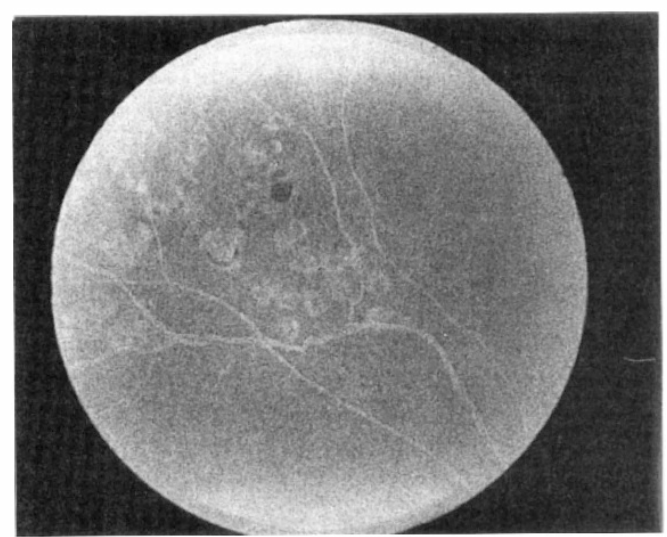

Fig. 4c.

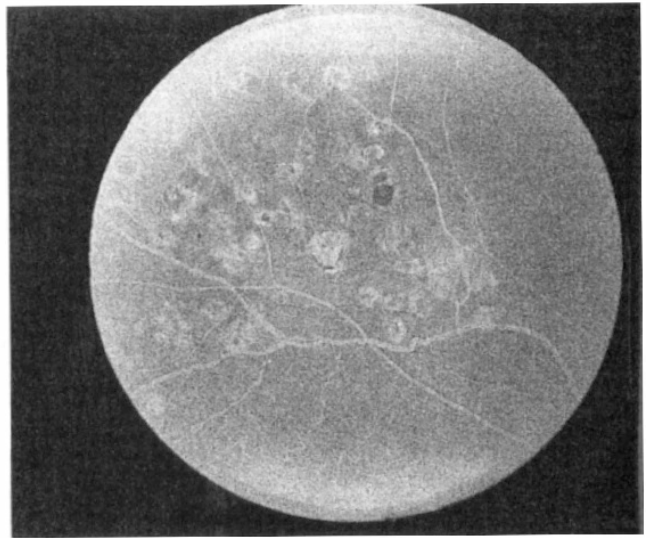

Fig. $4 b$.

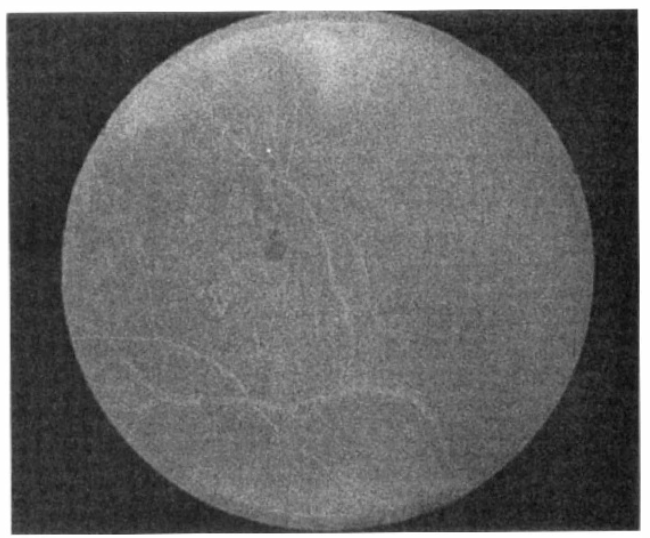

Fig. 4d.

Fig. 4. Fluorescein angiograms in a patient with Behçet's disease who has suffered recurrent branch retinal vein occlusion and subsequently developed retinal new vessels.

Figure 4 a (top left): late phase sodium fluorescein angiogram demonstrating profuse leakage from retinal new vessels. Figure $4 b$ (top right): 10 minute angiogram following injection of FD-4. Leakage of dye is seen from the new vessels. Figure 4c (bottom left): 10 minute angiogram following injection of FD-20. No leakage of dye is observed. Figure 4d (bottom right): 10 minute angiogram following injection of FD-150. No leakage of dye is observed.

tran angiography in man is a safe and feasible technique. The angiograms obtained were of sufficient quality to be used to determine the molecular size of leakage from diseased retinal vessels at various sites in eyes with posterior uveitis. FITC-dextrans are chemical conjugates of fluorescein and dextran, both of which are widely used in medicine with an acceptable incidence of adverse effects. Thus it is not surprising that, given the stringent quality control of the finished product undertaken in this study, there should have been no systemic toxicity observed after intravenous injection of any of the FITC-dextrans. Although under experimental conditions some FITC-dextrans may enhance leucocyteendothelial adhesion, ${ }^{8}$ there was no evidence of induction or progression of retinal inflammation in any of the subjects studied. These compounds have previously been shown to be stable in aqueous solution, ${ }^{9}$ in plasma and in vivo ${ }^{11}$ and the retinal vascular leakage observed in this study can therefore be confidently assigned molecular size values corre- 
sponding to the molecular weights of the substances injected (see Table II).

We observed FITC-dextrans of up to 150 $\mathrm{kDa}$ leaking from inflamed optic discs and of up to 20-kDa into areas of macular oedema, whereas only sodium fluorescein and 4-kDa leaked from areas of retinal neovascularisation. Due to the spectrum of disease in the patients selected for this study we were unable to give reliable estimates of leakage from areas of perivasculitis. Thus the molecular size of leakage from the optic disc in our patients (150-kDa: EDR $8.5 \mathrm{~nm}$ ) corresponds to the size of an immunoglobulin $\mathrm{G}$ molecule (160-kDa) whereas that seen at the macula (20-kDa: EDR $3.2 \mathrm{~nm}$ ) corresponds more closely to the size of $\beta_{2}$-microglobulin (ca 20$\mathrm{kDa}$ ). However, size of leakage from retinal new vessels (4-kDa: EDR $1.3 \mathrm{~nm}$ ) is so small that only small molecules are likely to be able to penetrate the perivascular space in such areas.

Using S-antigen-induced EAU in the rat as a model of human uveitis, ${ }^{11}$ we have also found FITC-dextrans of up to 20-kDa to leak from areas of perivasculitis and optic disc swelling (unpublished data). Comparable findings have been described in IRBPinduced experimental autoimmune uveoretinitis (EAU) in primates, ${ }^{8}$ where the optic discwas shown to leak 150-kDa FITC-dextran and peripheral retinal vessels $70-\mathrm{kDa}$ molecules. Similarly, FITC-dextrans of $3-\mathrm{kDa}$ and 20-kDa have been shown to leak from retinal vessels of rats with urethane or phototoxic retinopathy, ${ }^{11}$ and $40-\mathrm{kDa}$ FITCdextrans did not leak.

Several explanations might be professed to interpret the cellular pathology of FITC-dextran leakage at sites such as the optic disc and macular in posterior uveitis. Tight retinal endothelial junctions ${ }^{4.12}$ might be severely dis-

Table III Summary of leakage of FITC-dextrans at three retinal sites in patients with posterior uveitis

\begin{tabular}{lccc}
\hline Preparation & Disc & Macula & New vessels \\
\hline NaFl & $4 / 6$ & $5 / 6$ & $1 / 6$ \\
FD-4 & $3 / 6$ & $4 / 6$ & $0 / 6$ \\
FD-20 & $3 / 6$ & $5 / 6$ & $0 / 6$ \\
FD-150* & & $0 / 4$ & $0 / 4$ \\
\hline (4 patients) & $2 / 4$ & $0 / 4$ \\
\hline
\end{tabular}

*Not available for 2 patients rupted allowing the free intercellular diffusion of FITC-dextrans across the BRB at a rate proportional to their molecular weight, ${ }^{5.6 .13}$ thereby accounting for the intense peripapillary fluorescence seen in our study with sodium fluorescein compared with the relatively low fluorescence seen with FD-150. Alternatively, large molecules may move across the endothelium through intracellular fenestrations and channels, or by vesicular transport. Such a transport system has been demonstrated to occur for glycogen $(75-\mathrm{kDa})$ and dextran (250-kDa) in intestinal capillaries where the molecules preferentially reach the pericapillary space via plasmalemmal vesicles rather than by diffusion through the intercellular junctions of these physiologically permeable vessels. ${ }^{1+}$

Little ultrastructural evidence exists as to the behaviour of retinal endothelial junctions in inflammation. Lightman et al. ${ }^{6}$ were able to show in their primate model that leakage of large molecules (FD-150) was associated with breakdown of tight junctions between retinal endothelial cells. Areas that leaked smaller molecules (FD-70 or NaFl) showed no morphological changes in junctions and they concluded that the enhanced vascular permeability to smaller molecules was due to an increase in transcellular transport. Since our work broadly concurs with theirs it seems reasonable to suggest that similar mechanisms are operating in man.

A hypothesis might therefore be constructed to explain this differential breakdown in the BRB based on the physiological ability of the retinal vascular endothelial cell to exclude large molecular weight proteins. Both $\mathrm{NaFl}$ and FD-4 were observed to leak from areas of neovascularisation. These new vessels are fenestrated but may have relatively normal endothelial cells lining them. Thus they may be able to exclude larger proteins (FD-20 and FD-150) whilst the smaller ones can escape either by enhanced transcellular transport or by passive diffusion across fenestrae. A similar situation is envisaged in macular oedema but the exclusion mechanisms of the endothelial cells are further compromised so that molecules of up to 20 -kDa can get across. Finally, at the optic disc which leaked dextrans of all molecular weights, there may be a 
breakdown of the interendothelial junctions as well as compromised endothelial function. It is known that these changes are reversible as shown by the change in standard fluorescein patterns during treatment of posterior uveitis but whether the differential pattern of leakage in macula oedema for instance has prognostic value awaits to be determined.

In conclusion, differential retinal leakage of graded FITC-dextrans in uveitis patients appears to give a useful estimate of the size of plasma protein able to leak at sites of retinal inflammation. The technique might also be reasonably expected to measure the functional state of the retinal endothelium in such eyes, and therefore FITC-dextran angiography might usefully provide an objective measure of disease activity and subsequent prognosis in such patients.

We would like to thank Mr M Stanford for reviewing the manuscript, $\mathrm{Mr}$ Colin Clements for his expert photographic assistance, and Mrs D Paterson for secretarial help.

EGA was an MRC Training Awards Fellow and the work was supported by grants from the Iris Fund for the Prevention of Blindness. BAE was supported by a grant from the Special Trustees of St Thomas' Hospital.

Key words: FITC-dextran; fluorescein angiography; posterior uveitis.

\section{References}

${ }^{1}$ Graham EM, Stanford MR, Sanders MD, Kasp E, Dumonde DC: A point prevalence study of 150 patients with idiopathic retinal vasculitis: 1 . Diagnostic value of ophthalmological features. $\mathrm{Br} J$ Ophthalmol 1989, 73: 714-21.

${ }^{2}$ Dumonde DC, Kasp-Grochowska E, Graham EM, Sanders MD, Faure JP, DeKozak Y, Tuyen VV: Antiretinal autoimmunity and circulating immune complexes in patients with retinal vasculitis. Lancet 1982; 2: 787-92.
${ }^{3}$ Chioralia G, Salinen L, Baurmann H, Kremer F: Fluorescein-labelled dextrans as tracer substance for experimental angiograms. Acta Ophthalmologica 1976, 54: 665-7.

${ }^{4}$ Bellhorn RW: Permeability of blood-ocular barriers of neonatal and adult cats to fluorescein-labelled dextrans of selected molecular sizes. Invest Ophthalmol Vis Sci 1981; 21: 282-90.

${ }^{5}$ Rabkin MD, Bellhorn MB, Bellhorn RW: Selected molecular weight dextrans for in vivo permeability studies of rat retinal vascular disease. Exp Eye Res 1977; 24: 607-12.

${ }^{6}$ Lightman SL, Caspers-Velu LE, Hirose S, Nussenblatt RB, Palestine AG: Angiography with fluorescein-labelled dextrans in a primate model of uveitis. Arch Ophthalmol 1987; 105: 844-8.

${ }^{7}$ Bradford MM: A rapid and sensitive method for the quantitation of microgram quantities of protein utilising the principle of protein-dye binding. Analyt Biochem 1976; 72: 248-54.

${ }^{8}$ Gawlowski DM, Harding NR, Granger HJ: Leucocyte phagocytosis and alterations in microvascular integrity elicited by FITC-dextran 150 and epi-illumination in the microcirculation of the hamster cheek pouch. Microvas Res 1989; 37: $1-15$.

${ }^{y}$ de Belder AN and Granath K: Preparation and properties of fluorescein-labelled dextrans. Carbohydrate Res 1973; 30: 375-8.

${ }^{11}$ Schroder U, Arfors KE, Tangen O: Stability of fluorescein labelled dextrans in vivo and in vitro. Microvascular Res 1976; 11: 33-9.

${ }^{11}$ Stanford MR, Graham EM, Kasp E, Brown EC, Dumonde DC, Sanders MD: Retinal vasculitis: correlation of animal and human diseae. Eye 1987; 1: 69-77.

${ }^{12}$ Cunhas-Vas JG, Shakib M, Ashton N: Studies on the permeability of the blood-retinal barrier.I: On the existence, development, and site of a bloodretinal barrier. Brit J Ophthalmol 1966; 50: 441-53.

${ }^{1.3}$ Garner A, Ashton N, Tripathi R, Kohner EM, Bullpitt CJ, Dollery CT: Pathogenesis of hypertensive retinopathy, an experimental study in the monkey. Brit J Ophthalmol 1975; 59: 3-44.

${ }^{14}$ Simionescu N, Simionescu M, Pallade GF: Permeability of intestinal capillaries: Pathway followed by dextrans and glycogens. J Cell Biol 1972; 53: $365-92$. 